\title{
Syntheses, structures, chiroptical and magnetic properties of chiral clusters built from Schiff bases: a novel [MnIIMnIII 6NaI 2] core
}

(1)

(1)

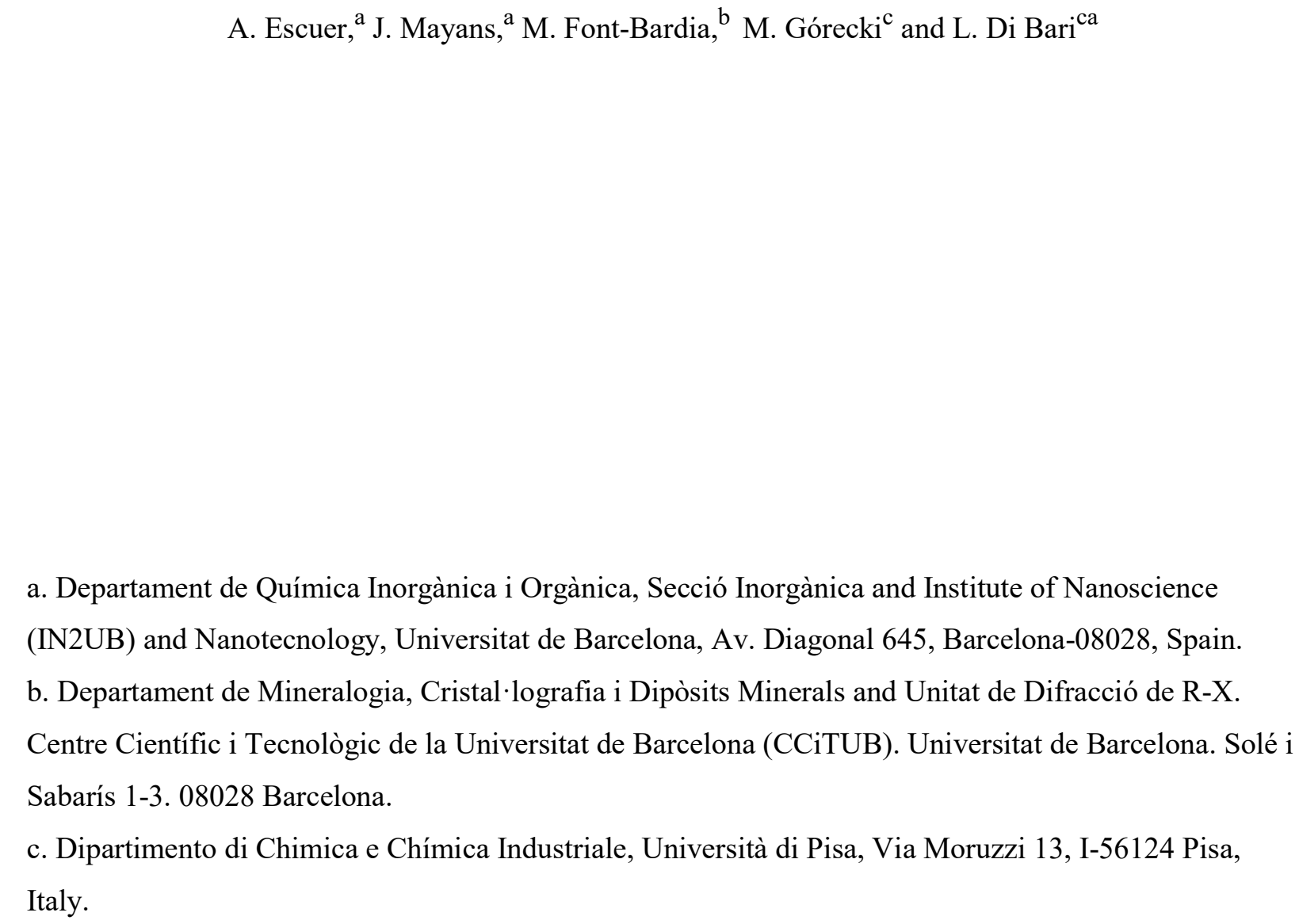


35 Chiral clusters with MnIIMnIII 3NaI and the new MnIIMnIII 6NaI 2 cores have been synthesised

36 employing enantiomerically pure Schiff bases and halide ligands. The new compounds have been

37 characterized by electronic circular dichroism (ECD) and magnetic susceptibility.

38

39

40 
Supramolecular chirality has received large attention along the last years in several research fields as chiral catalysis, recognition and sensing or CPL emission1 as well by its potentially different reactivity in biological environments.2 As a subfield, chiral self-assembled coordination compounds revealed to be attractive in the study of transfer of chirality, chiroptical properties (often lanthanide complexes), or magnetochemistry in close relationship to the search of chiral SMM or extended magnets.3 Employment of enantiomerically pure ligands allow the direct syntheses of chiral coordination compounds that can be useful in the search of multifunctional systems in which optical or emissive properties could be combined with its magnetic response. Among them, Schiff bases constitute an appropriate family of ligands to reach this target due to its ability to generate large nuclearity or high spin clusters and because these ligands can be easily functionalized to incorporate chiral centres. In this communication we report preliminary results from the employment of the R / S chiral Schiff base H2L obtained by condensation of o-vanilline and R- or S-phenylglycinol in manganese chemistry and the characterization of enantiomeric pairs of MnIIMn3 IIINaI penta and the new MnIIMn6 IIINa2 I nonanuclear complexes, in which the dianionic form of H2L links three different cations, Scheme 1.

Reaction of R- or S-H2L with manganese bromide and sodium azide yields the pentanuclear complexes [Mn4NaOL3Br4] [Mn4NaOL3Br4(H2O)]·[Mn4NaOL3Br3(MeOH)(MeCN)(H2O)]·[Mn4NaOL3Br3( $\mathrm{MeOH})(\mathrm{MeCN}) 2] \mathrm{Br} 2 \cdot 6 \mathrm{CH} 3 \mathrm{CN} \cdot 5 \mathrm{CH} 3 \mathrm{OH}(1 \mathrm{R})$ and $[\mathrm{Mn} 4 \mathrm{NaOL} 3 \mathrm{Br} 4(\mathrm{H} 2 \mathrm{O})] \cdot[\mathrm{Mn} 4 \mathrm{NaOL} 3 \mathrm{Br} 3(\mathrm{MeOH})(\mathrm{H} 2 \mathrm{O}) 2] \mathrm{Br} \cdot 6 \mathrm{CH} 3 \mathrm{CN} \cdot \mathrm{CH} 3 \mathrm{OH}(1 \mathrm{~S})$ which exhibit a core similar to some previously reported systems with azido or other ligands instead of halides and related Schiff bases. 4 In contrast, the reaction in basic $\mathrm{NaOH}$ medium yields the nonanuclear clusters [Mn7Na2(O)2L6X6]-solvents $(\mathrm{X}=\mathrm{Cl}, 2 \mathrm{R}$ and $2 \mathrm{~S}, \mathrm{X}=\mathrm{Br} 3 \mathrm{R})$, that exhibit an unprecedented core, (synthetic details in ESI). To our knowledge, the $\{(\mu 3-\mathrm{O}) \mathrm{MnIII} 3-\mathrm{MnII}-\mathrm{MnIII} 3(\mu 3-\mathrm{O})\}$ core has only been reported as fragment of larger Mn19 clusters5 and in one Mn7 system built from salicyloximate ligands.6 However, in this later case, the core was closer to two pseudocubanes sharing the central MnII cation and becomes less comparable The structures (ESI, Tables S1-S4) of 1R and 1S contain pentanuclear MnIIMn3IIINaI discrete units linked by three L2-ligands. The metallic cations determine a trigonal bipyramidal arrangement with the three $\mathrm{MnIII}$ ions in the equatorial plane and the MnII and NaI cations in the apical positions, Fig 1. The O-phenoxide and O-methoxide donors form a octahedral cavity that holds the $\mathrm{NaI}$ cation, which is bridged by means of the O-phenoxides to the MnIII cations whereas the O-alcoxide donors link the MnIII cations with the MnII ions. An additional $\mu 3-\mathrm{O}$ donor is placed in the centre of the triangle determined by the trivalent manganese cations. Noteworthy, the complicated structure of 1R contains four different clusters that differ in how the coordination sphere of the MnII cation is fulfilled: three fac-coordination sites are occupied in all cases by three $\mathrm{O}$-alcoxide donors from the L2- ligands but the remaining positions can be occupied by one bromide (tetracoordinated), three solvent molecules (hexacoordinated) or by one water molecule and one bromo ligand (pentacoordinated). For $1 \mathrm{~S}$ a similar fact was observed with the presence of two 
independent units with the MnII cations in octahedral or pentacoordinated environments (ESI, Fig. S2). The main structural consequence consists in the increase of the MnIII-O-MnII bond angles, which are close to $122^{\circ}$ (octahedral MnII), $118^{\circ}$ (pentacoordinated MnII) and $111^{\circ}$ (tetrahedral MnII). Remaining angles or distances of the cluster core are similar in all units (ESI, Table S2). The chirality transfer for complexes $1 \mathrm{R}$ and $1 \mathrm{~S}$ can be observed at level of the inner $\mathrm{NaI}$ cations that exhibit $\Lambda(1 \mathrm{R})$ or $\square(1 \mathrm{~S})$ configuration or at the whole helical shape of the molecule due to the tilted arrangement of the ligands with respect to the main axis of the molecule, Fig. 2. The R and S pairs of enantiomers of 2 consist of two pentanuclear units (similar to those described for 1), sharing the MnII cation, resulting a nonanuclear MnIIMn6 IIINa2I core, Fig. 3. The 22 positive charges are balanced by the six dianionic Schiff bases, six halides and two $\mu 3$-oxo donors. The L2ligands and the MnIII cations in each pentanuclear subunit are related by one C3 axis (R3 space group) but the lack of symmetry between them determine different bond parameters around Mn3 in each subunit: Mn2-O4-Mn3 bond angles takes a mean value of $125.8(3)^{\circ}$ whereas $\mathrm{Mn} 1-\mathrm{O} 1-\mathrm{Mn} 3$ is $122.9(3)^{\circ}$. Surprisingly, the helicity of each MnIIMn3IIINaI moiety turns in opposite sense and thus, the NaI cations show also opposite $\Lambda / \square$ configuration. Complex $3 R$, prepared to check if this structure can be extended to other halides, exhibits the same core than $2 \mathrm{R}$ and with the logical exception of the larger MnIII-Br bond distances, all bond parameters of the cluster core are very similar to 2 (ESI, Table S4). Noteworthy, in all cases the oxo ligand is slightly displaced out of the MnIII3 plane towards the NaI cation, with a Na-O distance of around $2.7 \AA$ and thus, the coordination of the sodium ions can be formally described as an apicated octahedron and the oxo donors can be assumed as $\mu 4-\mathrm{O}$ ligands. ECD spectra of the pairs of enantiomers $1 \mathrm{R} / 1 \mathrm{~S}$ and $2 \mathrm{R} / 2 \mathrm{~S}$ measured in solid phase and acetonitrile solution show perfect mirror images of each other, which confirm the enantiomeric purity of samples, Fig. 4. The spectra of the penta and nonanuclear complexes are closely related as can be expected for systems in which the ECD spectrum is directed mainly by chirality of the chelating ligands. Comparison between solid and solution spectra, in the 250-800 range suggest that the crystal structure is pretty close to the predominant conformer(s) in acetonitrile solution. As expected from the structural data the magnetic response for each kind of core is very similar and independent of the enantiomer or the halide donors. Thus, we provide a general description of their magnetic behaviour. The room temperature $\chi \mathrm{MT}$ value for the complexes with MnIIMn3 IIINaI core is close to $10.5 \mathrm{~cm} 3 \cdot \mathrm{mol}-1 \cdot \mathrm{K}$, slightly lower than the expected value for three isolated MnIII and one MnII cations $(13.375 \mathrm{~cm} 3 \cdot \mathrm{mol}-1 \cdot \mathrm{K})$ and this value decreases continuously on cooling down to a plateau between $25-5 \mathrm{~K}$, with a low $\mathrm{T}$ fall down to $6 \mathrm{~cm} 3 \cdot \mathrm{mol}-1 \cdot \mathrm{K}$ at $2 \mathrm{~K}$. In contrast, the complexes with MnIIMn6 IIINa2 I core exhibit a room temperature $\chi \mathrm{MT}$ value close to $19 \mathrm{~cm} 3 \cdot \mathrm{mol}-1 \cdot \mathrm{K}$, also lower than the expected value for six non-interacting MnIII and one MnII cations $(22.375 \mathrm{~cm} 3 \cdot \mathrm{mol}-$

$1121 \cdot \mathrm{K})$. On cooling, $\chi \mathrm{MT}$ decreases down to a well defined minimum around $120 \mathrm{~K}$ with a further increase 113 up to a maximum value close to $28 \mathrm{~cm} 3 \cdot \mathrm{mol}-1 \cdot \mathrm{K}$ at $10-15 \mathrm{~K}$ with a final decrease below these temperatures indicating a ferromagnetic response, Fig. 5. 
115 Previously reported related systems with MnIIMn3IIINaI core have different ligands in the elongated

116 coordination sites of the MnIII cations4 but in our case the clusters are close or possess an strict C3

117 symmetry with halide donors in these positions, simplifying the coupling scheme. Fit of the

118 experimental data was performed with PHI program7 applying a 2-J model in which J1 corresponds to

119 the interaction between the MnIII cations inside the triangular subunits and J2 corresponds to the

120 MnIII $\cdots$ MnII interactions according the Hamiltonians and coupling scheme shown in Fig. S3.

121 Excellent fits were obtained with consistent FM coupling for the MnIII-MnIII interactions (J1 in the

$122+0.8 /+0.9 \mathrm{~cm}-1$ range) and AF coupling for the MnII-MnIII interactions ( $\mathrm{J} 2$ in the $-2.3 /-3.7 \mathrm{~cm}-1$ range)

123 and $g$ around 1.9 .

124 Noteworthy, the new Mn7 core of 2-3 is similar to the central fragment of the $\mathrm{S}=83 / 2$ ground state

125 supertetrahedral vertexsharing Mn19 clusters, Fig. S4.5 In contrast with 2-3, the MnII-OMn III

126 interaction for $\mathrm{Mn} 19$ was ferromagnetic contributing to reach the maximum spin. Close inspection of the

127 bond parameters for both systems show that the MnII-O-MnIII bond angle for 2-3 is much larger than

128 for $\operatorname{Mn} 19\left(\approx 124^{\circ}\right.$ vs. $\left.\approx 110^{\circ}\right)$, and consequently the MnII $\cdots$ Ooxo distance increases by about $0.75 \AA$ and

129 the MnII environment for 2-3 becomes closer to a regular octahedron instead of the pseudo

130 octacoordination observed in Mn19, resulting in interactions of opposite sign. The easy axis of the MnIII

131 cations lies in the $\{\mu 3 \mathrm{O}-\mathrm{Mn} 3 \mathrm{III}\}$ plane directed towards the chloro ligands forming angles of around

$13260^{\circ}$ between them. This easy axis arrangement is unfavourable to reach high anisotropy and, as was

133 experimentally checked, no out-of-phase response was found in AC experiments. 
135 CONCLUSIONS

136

137 Reaction of manganese halides with enantiomerically pure Schiff bases obtained from the condensation

138 of o-vanilline and (R) or (S)-phenilglycinol lead to the characterization of chiral penta and nonanuclear

139 complexes with MnIIMn3 IIINaI and the rare MnIIMn6 IIINa2 I cores. Current efforts to fully

140 characterize series of related complexes changing the NaI cations by other monovalent or divalent

141 cations, its role as oxygen evolving systems and a wide magnetostructural correlation are in due course

142 and will be reported in a forthcoming full paper.

143 


\section{ACKNOWLEDGEMENTS}

145

146 Founds from Ministerio de Economía y Competitividad, Project CTQ2015-63614-P are acknowledged.

147 MG thanks for support from the Polish Ministry of Science and Higher Education ("Mobilnosc Plus"

148 grant no. 1286/MOB/IV/2015/0).

149 
1 M. Liu, L. Zhang and T. Wang, Chem. Rev. 2015, 115, 7304.

153

R. Carr, N. H. Evans and D. Parker, Chem. Soc. Rev., 2012, 41, 7673.

154

J. Crassous, Chem. Soc. Rev., 2009, 38, 830; b) H. Miyake, Symmetry 2014, 6, 880.

a) L.-L. Fan, F.-S. Guo, L. Yun, Z.-J. Lin, R. Herchel, J.-D. Leng, Y.-C. Ou and M.-L. Tong, Dalton Trans., 2010, 39, 1771; b) S. Nayak, H. P. Nayek, S. Dehnen, A. K. Powell and J. Reedijk, Dalton Trans., 2011, 40, 2699; c) P.-P. Yang, C.-Y. Shao, L.-L. Zhu and Y. Xu, Eur. J. Inorg. Chem., 2013, 5288; d) P.-P. Yang, L.-L. Zhu and Y. Xu, Z. Anorg. Allg. Chem., 2013, 639, 1821; e) C. Ding, C. Gao, S. Ng, B. Wang and Y. Xie, Chem. Eur. J., 2013, 19, 9961; L. Cong, X. Qin, W. Sun, Y. Wang, S. Ding and Z. Liu, New J. Chem., 2014, 38, 545; f) B. Gole, K. C. Mondal and P. S. Mukherjee, Inorg. Chim. Acta, 2014, 415, 151; Y. Song, G. Zhang, X. Qin, Y. Gao, S. Ding, Y. Wang, C. Du and Z. Liu, Dalton Trans., 2014, 43, 3880. Powell, Angew. Chem., Int. Ed., 2006, 45, 4926 ; b) S. Nayak, M. Evangelisti, A. K. Powell and J. Reedijk, Chem. Eur. J., 2010, 16, 12865; J.-L. Liu, J.-D. Leng, Z. Lin and M.-L. Tong, Chem. Asian J., 2011, 6, 1007; c) A. M. Ako, Y. Lan, O. Hampe, E. Cremades, E. Ruiz, C. E. Anson and A. K. Powell, Chem. Commun., 2014, 50, 5847.

N. F. Chilton, R. P. Anderson, L. D. Turner, A. Soncini and K. S. Murray, J. Comput. Chem., 2013, 34, 1164 


\section{Legends to figures}

Scheme 1 Coordination to one MnIII, one MnII and one NaI cations found in complexes 1- 3 for the L2ligand employed in this work.

Figure. 1 Left, view of one of the non equivalent clusters of 1R. Right, partially labelled common core of complexes 1R and 1S. Color code: MnIII, dark green; MnII, orange; NaI, light blue; N, navy; O red; $\mathrm{Br}$, firebrick..

Figure. 2 Left, a spacefill view of the two pentanuclear enantiomers of $1 \mathrm{R}$ and $1 \mathrm{~S}$ showing the helical arrangement of the ligands. Right, NaI environment for $1 \mathrm{R}(\Lambda)$ and $1 \mathrm{~S}(\square)$ viewed along the $\mathrm{O} 10-\mathrm{Na}$ 185 direction.

Figure. 3 Top, a view of cluster 2. Bottom, partially labelled common core of complexes 2R, 2S. Color code: as Fig.1; $\mathrm{Cl}$, violet.

189

190

Figure. 4 Solid state (left) and acetonitrile solution (right) ECD spectra for the pair of enantiomers of the MnIIMnIII 3NaI complexes 1 (S green line; R orange line) and MnIIMn6 IIINa2 I complexes 2 (S red line; R blue line, X10).

Figure 5. Plot of $\chi \mathrm{MT}$ product vs. T for one of the enantiomers of complexes 1S (diamonds, red axis), 


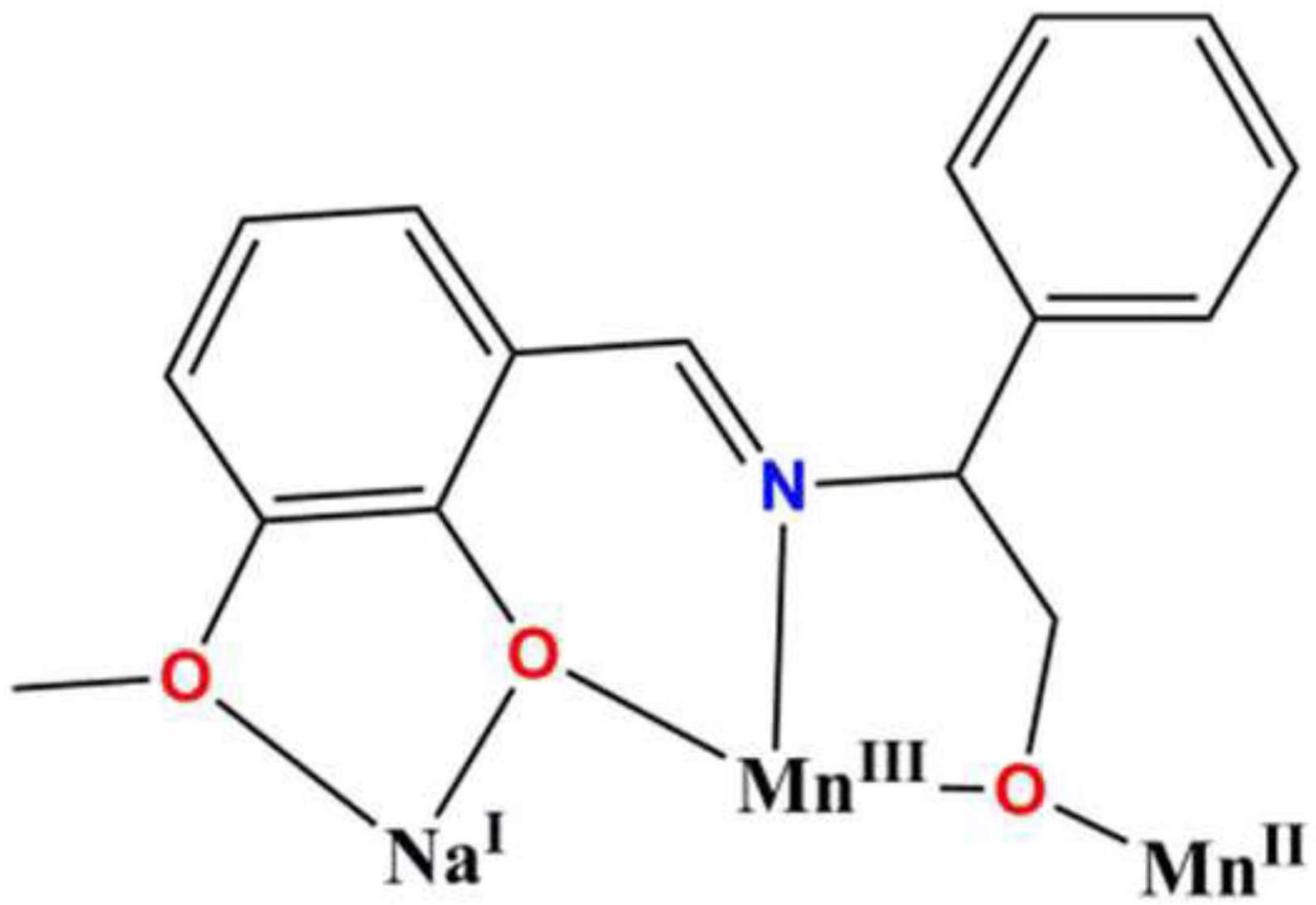


FIGURE 1

205

206
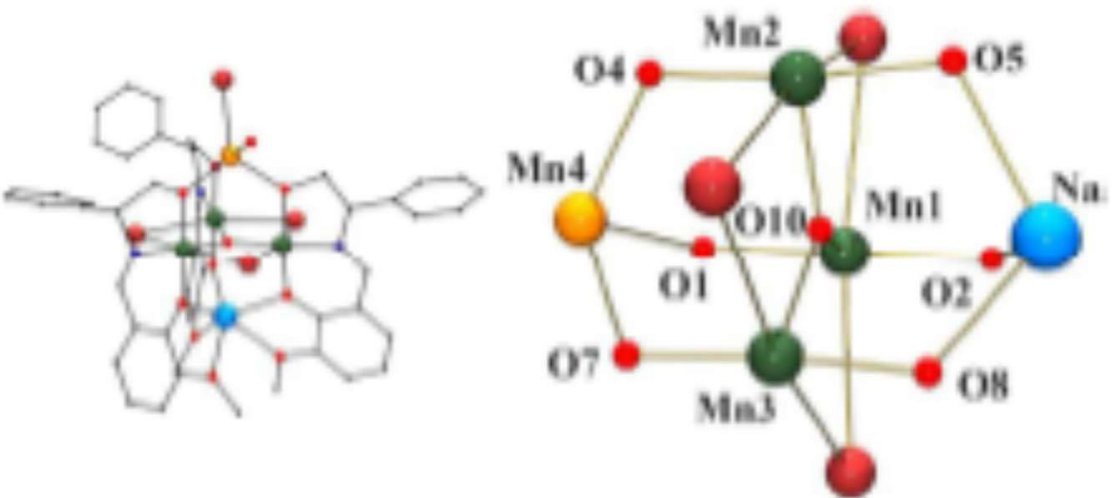

207

208 
FIGURE 2

210

211

212
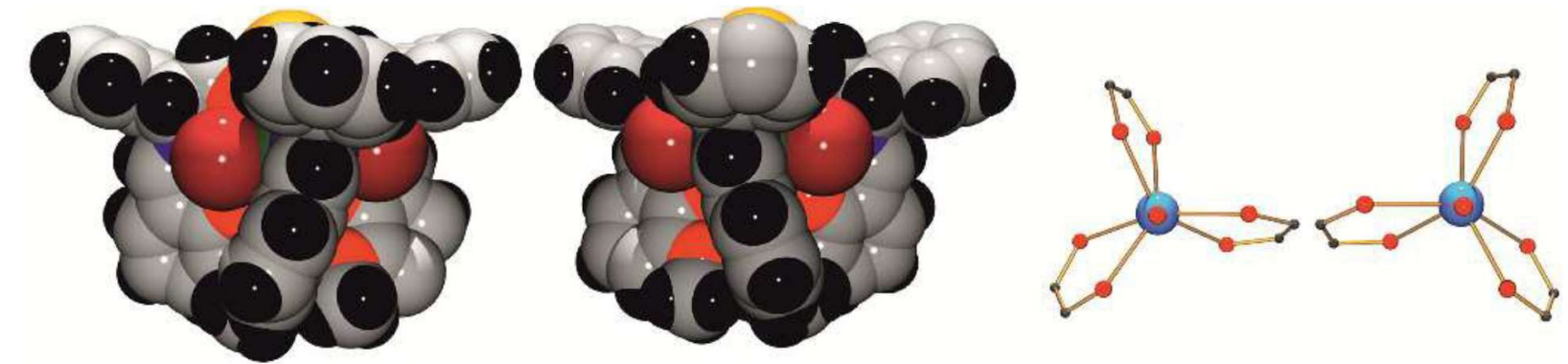

213 

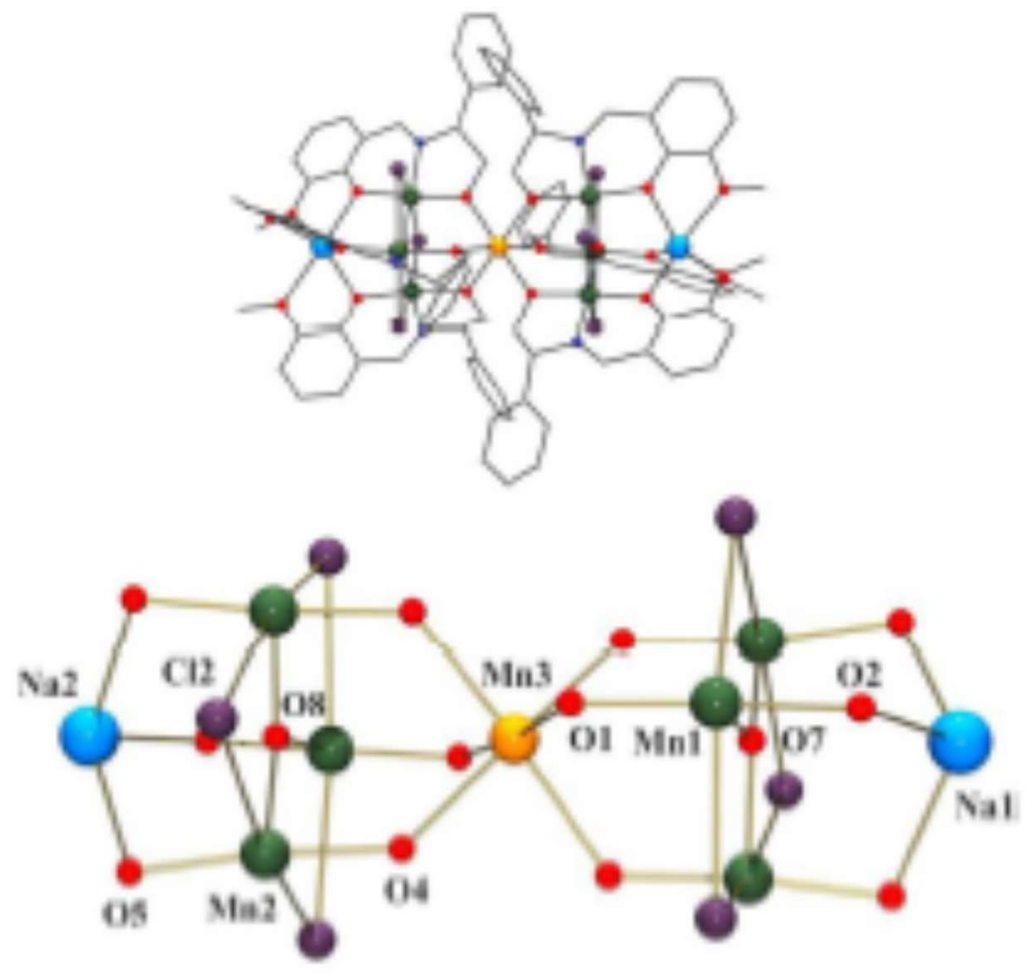

219 
FIGURE 4
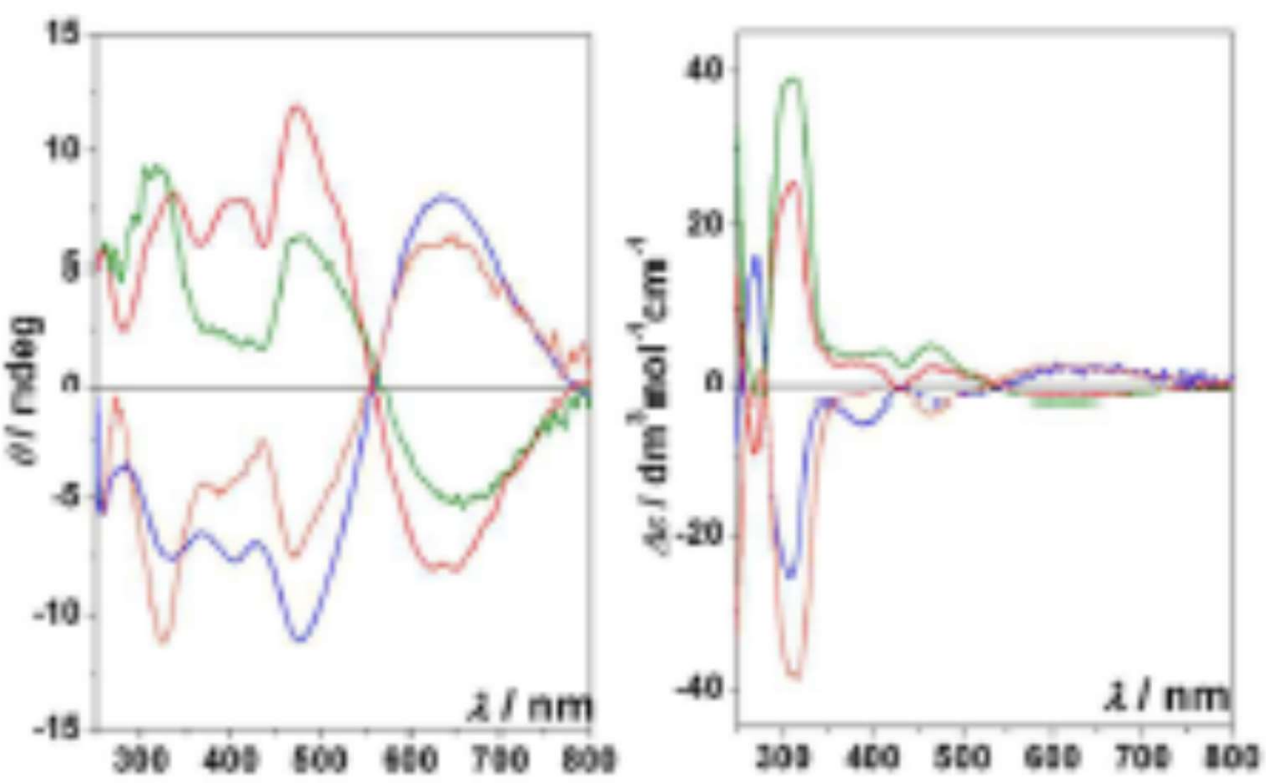

223

224 


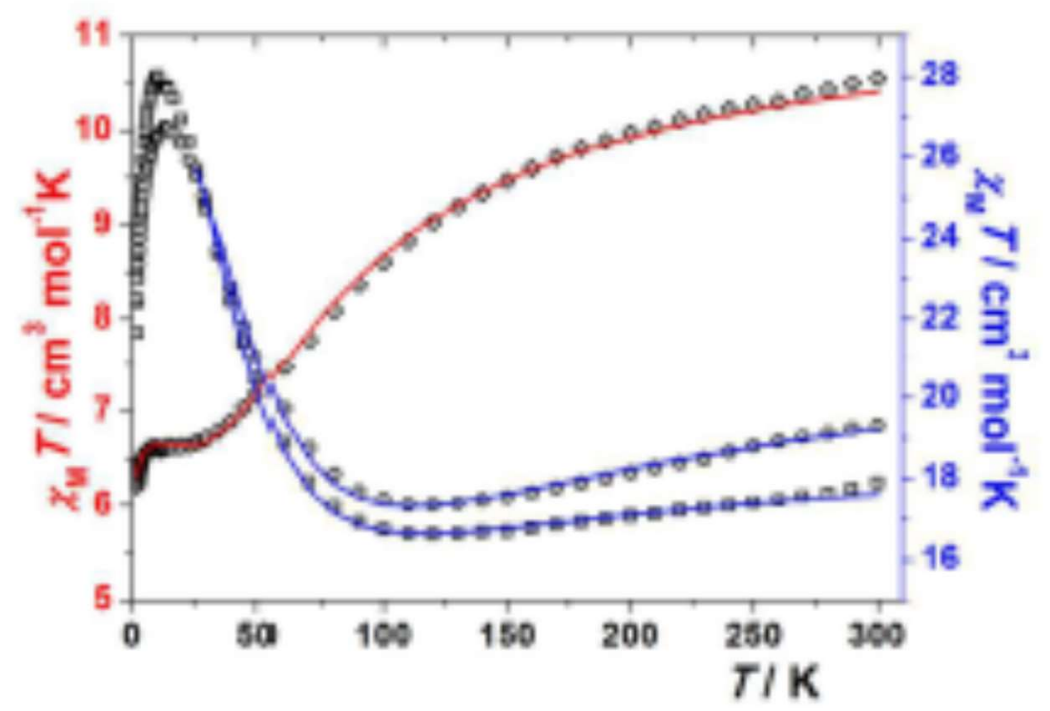




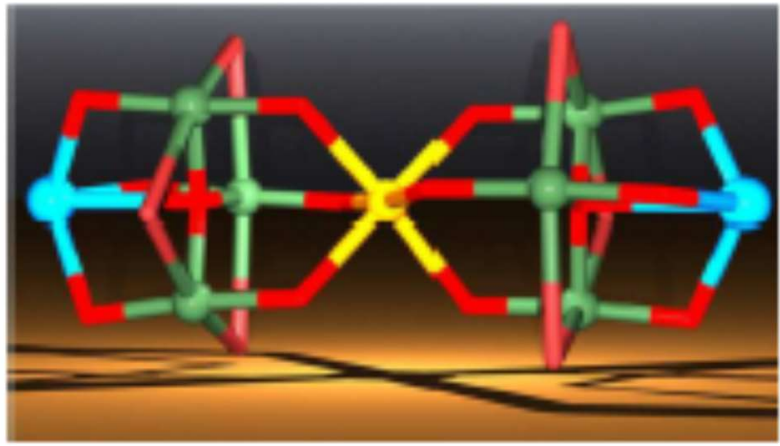

Enantiomeric pairs of clusters with MnIIMnIII3NaI and the unprecedented MnIIMnIII6NaI 2 cores have been synthesized employing enantiomerically pure Schiff bases. 